Bernard G. Fikkers

Marieke Staatsen

Frank J. A. van den Hoogen

Johannes G. van der Hoeven

\section{Early and late outcome after single step dilatational tracheostomy versus the guide wire dilating forceps technique: a prospective randomized clinical trial}

Received: 22 July 2010

Accepted: 18 March 2011

Published online: 12 April 2011

(C) The Author(s) 2011. This article is published with open access at Springerlink.com

B. G. Fikkers ( $)$ - J. G. van der Hoeven Department of Intensive Care Medicine, Radboud University Nijmegen Medical Centre, P.O. Box 9600,

6500 HB Nijmegen, The Netherlands e-mail: b.fikkers@ic.umcn.nl

Tel.: +31-24-3617273

Fax: +31-24-3541612

\section{Staatsen}

Department of Anesthesiology,

Radboud University Nijmegen Medical

Centre, P.O. Box 9600, 6500 HB Nijmegen,

The Netherlands

\section{F. J. A. van den Hoogen}

Department of Otorhinolaryngology,

Radboud University Nijmegen Medical

Centre, P.O. Box 9600, 6500 HB Nijmegen,

The Netherlands

\begin{abstract}
Purpose: Percutaneous tracheostomy is frequently performed in long-term ventilated patients in the intensive care unit (ICU). Unfortunately, despite many years of experience, the optimal technique is still unknown, especially considering the occurrence of late complications. The purpose of this study was to determine which of the two most frequently used percutaneous tracheostomy techniques performs best with the emphasis on late complications. Methods: This prospective randomized trial involved 120 patients, comparing two techniques of percutaneous tracheostomy, the guide wire dilating forceps (GWDF) and the single step dilatational tracheostomy (SSDT) technique. Results: Sixty patients in each group underwent a percutaneous tracheostomy and were followed for up to 3 months after decannulation. The majority of complications in both groups were minor (58.3\% in the GWDF group and
\end{abstract}

$61.7 \%$ in the SSDT group). We found a trend towards more major perioperative complications in the GWDF group versus the SSDT group, 10.0 versus $1.7 \%(p=0.06)$. One patient in the SSDT group developed a significant tracheal stenosis. However, this may also have been related to prolonged translaryngeal intubation. Results of magnetic resonance imaging (MRI) investigations showed only minor tracheal changes. Only $37.5 \%$ of patients in the GWDF group and $31.8 \%$ in the SSDT group had no complaints after their percutaneous tracheostomy. Conclusion: Compared with the GWDF, the SSDT shows a trend toward less major perioperative complications with a comparable long-term outcome.

Keywords Percutaneous . Tracheostomy - Guide wire dilating forceps - Single step tracheostomy . Complications

\section{Introduction}

Percutaneous tracheostomy is a common procedure in patients on the intensive care unit (ICU) who need prolonged mechanical ventilation, airway suctioning, or have an obstruction of the upper airway [1,2]. The two most widely used techniques are the guide wire dilating forceps (GWDF) and the single step dilatational tracheostomy (SSDT) $[3,4]$. Only two prospective randomized trials have been published comparing these techniques; however, these trials were small, were not adequately randomized, or did not investigate late complications [5, 6]. Previously, we retrospectively compared these techniques in 342 patients, and concluded that they were comparable [7].

The reason that so few studies analyzed late complications may be because follow-up of these patients can be difficult because of physical and psychological restraints $[5,8,9]$. Another problem is the lack of standardized 
outcome definitions and the way in which late complications have been examined [10]. The current trial was set up to compare prospectively the GWDF and SSDT techniques, with special attention to late complications. To date, this question has not been adequately addressed.

\section{Patients and methods}

This prospective randomized trial was conducted in the 31-bed intensive care unit of the Radboud University Nijmegen Medical Centre. Our medical ethics committee approved the protocol. The trial was preregistered under ClinicalTrials.gov Identifier NCT 00184925. Patients with an indication for tracheostomy were included after written informed consent was obtained from their family members or occasionally from the patient [11]. Randomization was done by way of sealed closed envelops by an independent statistician. Exclusion criteria for percutaneous tracheostomy were anatomical abnormalities like goiter, no palpable landmarks, distance from cricoid to manubrium less than $3 \mathrm{~cm}$ at maximum extension, infection at the intended tracheostomy site, severe uncorrectable coagulopathy, positive end-expiratory pressure (PEEP) $>10 \mathrm{cmH}_{2} \mathrm{O}$, high intracranial pressure $\left(>20 \mathrm{cmH}_{2} \mathrm{O}\right)$, age less than 18 years, or body weight less than $40 \mathrm{~kg}$.

Between September 2003 and August 2006, we included a total of 120 patients. Sixty underwent a GWDF technique (SIMS Portex, Hythe, Kent, UK) and 60 a SSDT technique (Portex Ultraperc ${ }^{\mathrm{TM}}$, Smith Medical, Hythe, Kent, UK). The percutaneous tracheostomy was performed by an intensivist or intensive care fellow under close supervision according to a standard procedure, including bronchoscopic guidance in every patient, as described elsewhere [11, 12].

Complications were recorded as described previously [7, 13]. "Procedure time" is the time from incision of the skin until placement of the cannula. Ease of the procedure was graded on a numeric rating scale, where 0 indicated "extremely easy" and 10 "extremely difficult." "Perioperative complications" are complications occurring during the procedure or occurring in the first $24 \mathrm{~h}$ after the procedure. "Complications while cannulated" are defined as complications occurring between $24 \mathrm{~h}$ after the procedure until decannulation. "Late complications" are defined as complications occurring after decannulation. Complications were divided into minor, intermediate, and major. "Minor complications" are clinically irrelevant without patient harm. For example, a minor bleeding complication is a bleeding requiring local pressure, one suture and/or less than 100 -ml blood loss, or the occurrence of some intratracheal blood. "Intermediate complications" may potentially be harmful for the patient. "Major complications" require medical or surgical intervention and include bleeding requiring blood transfusion, perforation of the esophagus, or infection that needs treatment with antibiotics.

According to the study protocol, a chest X-ray was acquired $1 \mathrm{~h}$ after the procedure. General patient data recorded included age, sex, body mass index (BMI), indication for intensive care admission, indication for tracheostomy, days of intubation, and the SOFA score (Sequential Organ Failure Assessment).

Three months after decannulation, the larynx and trachea were evaluated by magnetic resonance imaging (MRI) scan of the neck using 4-mm slices, as described previously $[14,15]$.

Patients were also evaluated by an ear, nose, and throat (ENT) specialist, with special attention to scar and other possible tracheostomy-related problems. We performed fiberoptic tracheoscopy to evaluate vocal cord mobility and signs of airway obstruction. Preceding the ENT visit, patients were asked to fill in a questionnaire (Table 4).

Data were analyzed with Statistical Product and Service Solutions (SPSS) version 16.0. Data are given as mean \pm standard deviation (SD) or median (interquartile range, IQR). All variables were tested for normal distribution using the Kolmogorov-Smirnov test. Continuous variables were compared with the Student's $t$ test. For variables with non-normal distribution the Mann-Whitney test was employed. Categorical variables were compared with the chi-square test. A $p$ value of less than 0.05 was accepted as statistically significant. Analysis of covariance (ANCOVA) was used to correct for experience in relation to the procedure time.

\section{Results}

A flow diagram and summary of the study are shown in Fig. 1. Patient demographics are shown in Table 1. Reasons for intensive care admission and indications for tracheostomy were comparable between the two groups. An ICU fellow performed the majority of the procedures but always under close supervision of an intensivist (81.4\% SSDT and 67.2\% GWDF, $p=0.06$ ). The remaining procedures were done by an intensivist. No significant difference $(p=0.47)$ was found between the ease of both procedures, GWDF group median 3 (range 1.5-6) and SSDT group median 3 (range 2-4).

\section{Perioperative complications}

There was no significant difference between the two groups regarding minor perioperative complications: 35 complications in the GWDF group versus 37 in the SSDT group (Table 2). Nineteen patients had more than one minor perioperative complication. 
Fig. 1 Flow diagram and study summary

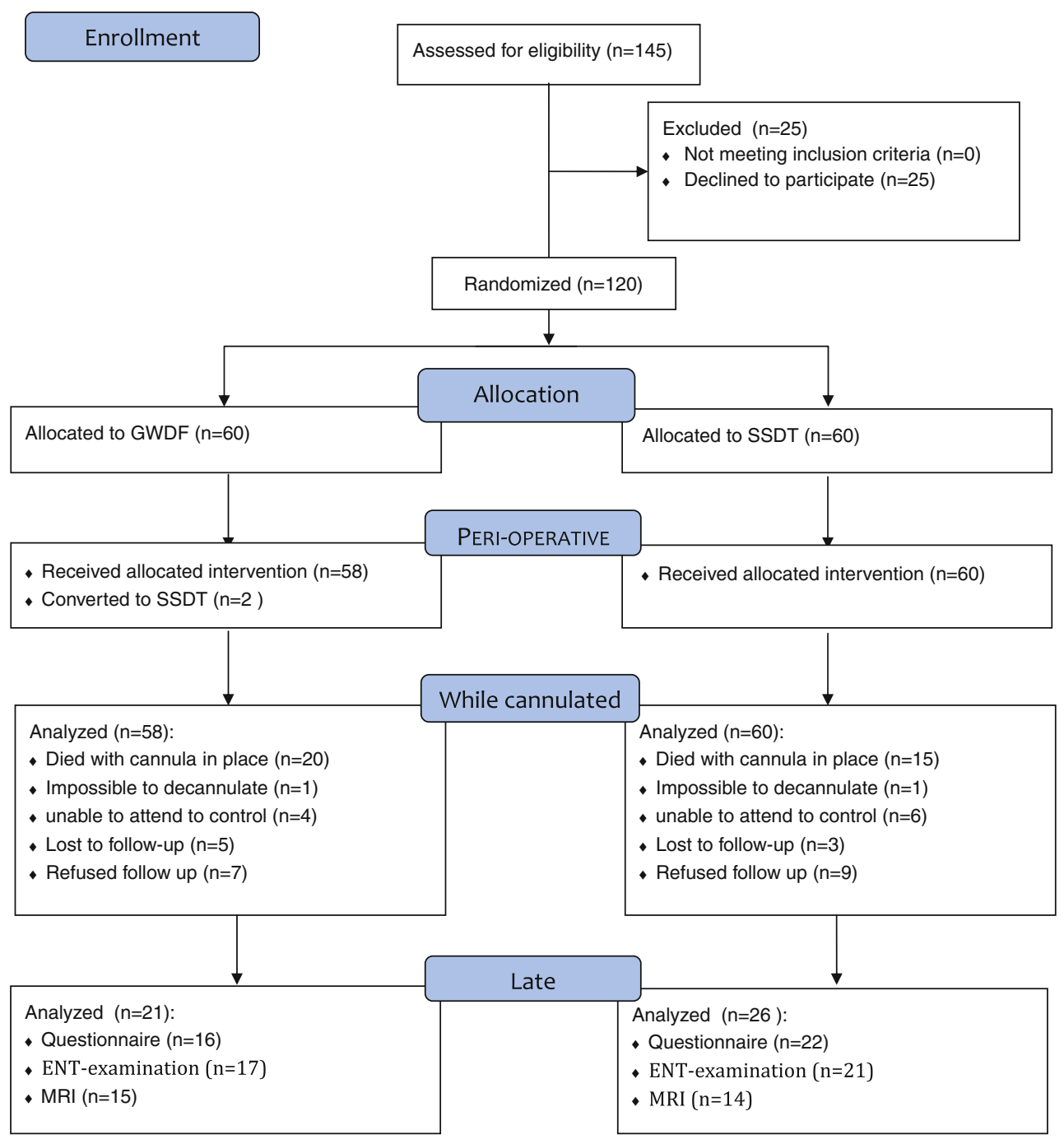

Table 1 Demographic data

\begin{tabular}{llll}
\hline & $\begin{array}{l}\text { GWDF } \\
n=60 \text { median } \\
{[\text { IQR 25-75] }}\end{array}$ & $\begin{array}{l}\text { SSDT } \\
n=60 \text { median } \\
{[\text { IQR 25-75] }}\end{array}$ & $p$ value \\
\hline Age (years) & $60[45-72]$ & $63[48-70]$ & 0.65 \\
Male (\%) & $39(65 \%)$ & $42(70 \%)$ & 0.35 \\
BMI & $25[23-27]$ & $25[24-27]$ & 0.50 \\
Procedure time (min) & $10[5-18]$ & $8[5-10]$ & 0.21 \\
Intubation time until tracheostomy (days) & $15[8-24]$ & $18[10-26]$ & 0.39 \\
Cannulation time (days) & $18[11-33]$ & $29[13-40]$ & 0.20 \\
LOS in ICU (days) & $28[20-51]$ & $30[21-50]$ & 0.70 \\
SOFA & $4[3-6]$ & $4[3-5]$ & 0.80 \\
\hline
\end{tabular}

LOS length of stay

There were seven major perioperative complications, the inability to introduce the cannula. These two six $(10 \%)$ in the GDWF group and one (1.7\%) in the patients were excluded from follow-up. One died SSDT group $(p=0.06)$. In the GDWF group three 23 days after percutaneous dilational tracheostomy major bleedings occurred and one pretracheal insertion. (PDT) and the other survived. Both had no complicaThe one major complication in the SSDT group was a tions related to the procedure. There were no signs of major bleeding. In two patients in the GWDF group, air leak or pneumothorax on the chest X-ray in any of the procedure was converted to an SSDT, because of the patients. 
Table 2 Perioperative complications

\begin{tabular}{llll}
\hline Complication & $\begin{array}{l}\text { GWDF } \\
n=60(\%)\end{array}$ & $\begin{array}{l}\text { SSDT } \\
n=60(\%)\end{array}$ & $p$ value \\
\hline No complications at all & $29(48.3)$ & $32(53.3)$ & 0.58 \\
Minor & $35(58.3)$ & $37(61.7)$ & 0.43 \\
Minor blood loss & $11(18.3)$ & $7(11.7)$ & 0.22 \\
Difficult puncture or punctured endotracheal tube & $8(13.3)$ & $6(10.0)$ & 0.39 \\
Puncture posterior tracheal wall (without emphysema) & $1(1.7)$ & $2(3.3)$ & 0.50 \\
Difficult dilation & $3(5.0)$ & $3(5.0)$ & 0.66 \\
Difficult cannula insertion (>2 attempts) & $2(3.3)$ & $0(0)$ & 0.25 \\
Hypoxemia shorter than 5 min (sat. <90\%) & $2(3.3)$ & $1(1.7)$ & 0.50 \\
Hypotension shorter than 5 min (systole <90 mmHg) & $1(1.7)$ & $8(13.3)$ & 0.02 \\
Tracheal ring fracture & $1(1.7)$ & $3(5.0)$ & 0.31 \\
Other complications & $6(10.0)$ & $7(11.7)$ & 0.50 \\
Intermediate & $1(1.7)$ & $3(5.0)$ & 0.31 \\
Hypotension or hypoxemia longer than 5 min & $1(1.7)$ & $3(5.0)$ & 0.31 \\
Major & $6(10.0)$ & $1(1.7)$ & 0.06 \\
Pretracheal insertion & $1(1.7)$ & $0(0)$ & 0.50 \\
Blood loss requiring blood transfusion & $3(5.0)$ & $1(1.7)$ & 0.31 \\
Conversion to alternate technique & $2(3.3)$ & $0(0)$ & 0.25 \\
Total one complication & $31(51.7)$ & $28(46.7)$ & 0.36 \\
Total two complications & $11(18.3)$ & $11(18.3)$ & 0.59 \\
Total three complications & $0(0)$ & $2(3.3)$ & 0.25 \\
Overall complication number & $42(70)$ & $41(68.3)$ & 0.50 \\
\hline
\end{tabular}

Complications while cannulated

A total of 118 patients were analyzed for complications while cannulated, 58 in the GWDF group and 60 in the SSDT group (Table 3). Eighty-four patients did not have any complication while cannulated, $45(77.6 \%)$ in the GWDF group versus 39 (65\%) in the SSDT group (NS). Thirty-four patients showed 39 complications while cannulated, $13(22.4 \%)$ patients in the GWDF group versus $21(35 \%)$ patients in the SSDT group $(p=0.08)$.

\section{Complications in GWDF group}

One complication was certainly related to the tracheostomy, although it is unlikely that it was related to the specific technique. This patient was admitted because of chronic respiratory insufficiency with morbid obesity (BMI $49 \mathrm{~kg} / \mathrm{m}^{2}$ ). Four days after admission and intubation, an uncomplicated PDT was performed. Three days later a fenestrated cannula was placed uneventfully, because of the strong desire of the patient to communicate. However, on the eighth day, she developed a stomal infection requiring antibiotic treatment. Despite this, the infection worsened. A CT scan showed tissue infiltration, but no abscess formation. Shortly after returning to the ICU, the cannula was dislocated and she had a difficult emergency re-intubation, because of massive obesity and swelling. The infection was treated with surgical debridement and antibiotics. After 5 weeks of endotracheal intubation, she underwent a surgical tracheostomy. Unfortunately, she died 12 weeks later, 144 days after admission to the ICU.
Table 3 Complications while cannulated

\begin{tabular}{llll}
\hline Complication & $\begin{array}{l}\text { GWDF } \\
n=58(\%)\end{array}$ & $\begin{array}{l}\text { SSDT } \\
n=60(\%)\end{array}$ & $p$ value \\
\hline Minor & $6(10.3)$ & $14(23.3)$ & 0.04 \\
Bleeding requiring local pressure & $4(6.9)$ & $8(13.3)$ & 0.18 \\
Stridor with empty cuff & $0(0)$ & $1(1.7)$ & 0.50 \\
Problems with swallowing & $0(0)$ & $1(1.7)$ & 0.50 \\
Infection & $0(0)$ & $2(3.3)$ & 0.25 \\
Granulation tissue around tracheostomy & $1(1.7)$ & $0(0)$ & 0.50 \\
Cuff leakage & $1(1.7)$ & $2(3.3)$ & 0.36 \\
Other complications & $3(5.2)$ & $5(8.3)$ & 0.36 \\
Major & $6(10.3)$ & $5(8.3)$ & 0.50 \\
Cannula obstruction & $4(6.9)$ & $3(5.0)$ & 0.50 \\
Accidental decannulation with problematic & $2(3.4)$ & $2^{\mathrm{a}}(3.3)$ & 0.69 \\
recannulation & & & \\
Total one complication & $13(22.4)$ & $21(35)$ & 0.08 \\
Total two complications & $2(3.4)$ & $3(5)$ & 0.50 \\
Overall complication number & $15(25.9)$ & $24(40)$ & 0.06 \\
\hline
\end{tabular}

${ }^{a}$ One patient had a significant subglottic stenosis 


\section{Complications in SSDT group}

None of the complications could be related to the procedure.

In one patient, a cannula change was complicated by pretracheal positioning of the cannula, and the patient was re-intubated. Subsequently, a bronchoscopy showed granulation tissue cranially of the cannula. The trachea was redilated and a cannula was placed uneventfully.

One patient underwent an uncomplicated PDT, 18 days after endotracheal intubation. After 7 weeks, she was decannulated, but unable to breathe spontaneously, because of upper airway obstruction and was recannulated immediately. Tracheoscopy showed diminished movements of the vocal cords. On CT scan, a subglottic stenosis $3 \mathrm{~cm}$ under the level of the glottis was seen, but also above the level of the tracheostomy and therefore may also have been caused by prolonged translaryngeal intubation. Eventually, this patient needed a tracheal resection.

\section{Late complications}

In the GWDF group 21 patients and in the SSDT group 26 patients were amendable for follow-up of late complications, see Fig. 1. Of those, 13 patients (GWDF) and 14 (SSDT) were able to take part in all follow-up examinations, i.e., MRI, ENT visit, and the questionnaire.

Thirty-eight patients completed the questionnaire, 16 patients from the GWDF and 22 from the SSDT group (Table 4). A total of $37.5 \%$ of patients in the GWDF group and $31.8 \%$ in the SSDT group had no complaints at all. Problems were mostly minor and not significantly different between groups. Nine patients (seven in the GWDF and two in the SSDT group) were dissatisfied with their scar $(p=0.02)$ and four requested a scar correction (three in the GWDF versus one in the SSDT group).

Table 4 Late complications questionnaire

\begin{tabular}{llll}
\hline & GWDF & SSDT & $p$ value \\
\hline Total number of questionnaires & 16 & 22 & 0.16 \\
No complaints & $6(37.5)$ & $7(31.8)$ & 0.49 \\
Sore throat & $0(0)$ & $3(13.6)$ & 0.18 \\
Speech difficulties & $9(56.2)$ & $10(45.4)$ & 0.37 \\
$\quad$ with change of voice & & & \\
Swallowing difficulties & $2(12.5)$ & $5(22.7)$ & 0.36 \\
Wheezing & $2(12.5)$ & $4(18.2)$ & 0.50 \\
Coughing (more than normal) & $5(31.2)$ & $8(36.4)$ & 0.51 \\
Shortness of breath & $5(31.2)$ & $5(22.7)$ & 0.41 \\
Scar problems & $7(43.7)$ & $2(9.1)$ & 0.02 \\
Painful & $1(6.3)$ & $1(4.5)$ & 1.0 \\
Cosmetical & $3(18.8)$ & $1(4.5)$ & 0.29 \\
Scar correction requested & $3(18.8)$ & $1(4.5)$ & 0.29 \\
$\quad$ by patient & & & \\
\hline
\end{tabular}

Table 5 Late outcome ENT visit

\begin{tabular}{lll}
\hline & $\begin{array}{l}\text { GWDF } \\
n=17(\%)\end{array}$ & $\begin{array}{l}\text { SSDT } \\
n=21(\%)\end{array}$ \\
\hline $\begin{array}{l}\text { Scar problems } \\
\text { Invisible scar }\end{array}$ & $6(35.3)$ & $12(57.1)$ \\
$\begin{array}{l}\text { Subtle, visible } \\
\text { Obvious visible but acceptable }\end{array}$ & $\begin{array}{l}10(58.8) \\
10(47.6)\end{array}$ & $5(23.8)$ \\
$\begin{array}{l}\text { Unaesthetic scar } \\
\text { Tracheoscopy }\end{array}$ & $3(17.6)$ & $0(0)$ \\
$\begin{array}{l}\text { Stenosis } \\
\text { Granulation tissue }\end{array}$ & $1(5.9)$ & $0(0)$ \\
$\begin{array}{l}\text { Other } \\
\text { Vocal cord mobility }\end{array}$ & $0(0)$ & $0(0)$ \\
$\begin{array}{l}\text { Normal } \\
\text { Moderately restricted/reduced } \\
\text { Seriously restricted/immobile }\end{array}$ & $1(5.9)$ & $1(4.8)$ \\
$\quad$ vocal cord & $15(88.2)$ & $18(85.7)$ \\
Follow-up ENT after 6 months & $0(0)$ & $1(4.8)$ \\
\hline
\end{tabular}

Thirty-eight patients were investigated by an ENT specialist (FvdH) 3 months after decannulation, 17 patients from the GWDF group and 21 from the SSDT group (Table 5). The ENT specialist concluded that in three patients the scars were indeed unaesthetic, and in those patients, all in the GWDF group, a correction was done. In three patients $(8 \%)$ abnormalities were seen by laryngotracheoscopic examination, two in the GWDF group and one in the SSDT group. One patient was admitted after a complicated coronary artery bypass grafting for which he was intubated for 6 weeks. On tracheoscopy, a subglottic tracheal stenosis was seen. Unfortunately, an MRI scan could not be performed because this patient had artificial ear ossicles. The relation between the complication and the percutaneous tracheostomy therefore remains unclear. In six patients (three in the GWDF group and three in the SSDT group), problems of vocal cord mobility were seen. In one there was minimal vocal cord impairment and in two patients, one of the vocal cords showed decreased motility, and in three patients one of the vocal cords was immobile.

\section{MRI investigation}

Thirty-four patients were investigated by using MRI. Three MRIs could not be analyzed because of technical problems, leaving 31 patients $(26 \%)$ for analysis. The precise position of the tracheostomy was difficult to see. Only 12 patients (39\%) showed minimal narrowing of the trachea. No severe stenosis was seen.

\section{Discussion}

To our knowledge, this is the largest prospective randomized trial comparing GWDF and SSDT. In general, 
no differences were found in complication rates. However, major perioperative complications tended to be higher in the GDWF group $(p=0.06)$. Three GWDF procedures (vs. one SSDT) resulted in a major bleeding and two GWDF procedures (in elderly patients) were converted to a SSDT. There was no procedure-related mortality.

By meticulously analyzing the procedure, we observed perioperative complications in almost half of the patients. Even though we feel that this observation is important in order to clarify the exact incidence of complications, only major complications result in clinical consequences. Few prospective trials comparing GWDF and SSDT have been done so far. Ambesh et al. [6], comparing GWDF and SSDT in 60 patients, found significantly more bleeding and difficult dilatation in the GWDF group, but did not favor any technique. Patients were followed throughout their hospital stay, and half of the patients could be decannulated. Only subjective complaints were evaluated. Three patients reported voice changes. No formal ENT or radiological follow-up was done. Añón et al. [5], comparing GWDF to SSDT in 53 patients, concluded that there were no differences between GWDF and SSDT, although in three (out of 26) GWDF tracheostomies, the procedure could not be finished. Seventeen out of 53 patients were evaluated for late complications, but only three (all belonging to the GWDF group) reported minor problems.

We observed a somewhat lower rate of tracheal ring fracture with the SSDT compared with the literature, 5 versus $8.9-36 \%$ [6, 16-18]. A possible explanation for this is that we used the Ultraperc ${ }^{\mathrm{TM}}$ instead of the Blue Rhino $^{\mathrm{TM}}$ set. When comparing these sets in a mannequin and in a porcine airway model, Patel et al. [19] reported that dilatation with the Ultraperc $^{\mathrm{TM}}$ required less force than with the Blue Rhino ${ }^{\mathrm{TM}}$. The significance of tracheal ring fracture is unknown. A recent series of tracheal ring fractures did not find an association with tracheal stenosis [20].

A chest X-ray after the procedure did not show any complication related to the procedure and therefore, in agreement with previous studies, we state there is no indication for a routine chest X-ray after an uneventful PDT [21]. Swallowing problems are common after endotracheal intubation [22]. Recently, it was shown that this may occur in up to $38 \%$ of patients after PDT [23]. We found an incidence of swallowing problems in $18.4 \%$ of patients. However, in order to adequately diagnose swallowing problems, fiberoptic endoscopic evaluation of swallowing (FEES) is necessary. We used a long-term follow-up by questionnaire, ENT examination, and MRI, which is probably less well suited to rule out swallowing problems. MRI may be particularly useful to diagnose or exclude a tracheal stenosis; however, the investigation is cumbersome in critically ill patients.

From the patients amendable to follow-up, only 13 (21.6\%) in the GWDF group and $14(23.7 \%)$ in the SSDT group fulfilled the whole follow-up protocol of this trial. According to the late complications questionnaire, only $37.5 \%$ in the GWDF group and $31.8 \%$ in the SSDT group had no complaints at all. The majority of reported complaints were minor. Moreover, it is difficult to distinguish between complaints (like voice problems or tracheal stenosis) that are really due to the tracheostomy or due to prolonged endotracheal intubation and/or underlying disease $[24,25]$.

The strength of this trial is that it is the largest prospective randomized trial so far comparing the most widely used percutaneous tracheostomy techniques. By standardizing definitions, we performed a meticulous observation of the procedure and postoperative follow-up. Also the fact that all our procedures were done with fiberoptic guidance may be of importance, because this facilitates optimal positioning of the cannula between the rings and in the midline. However, this trial also has several limitations. First, the study may have been underpowered to determine relevant differences between the two groups. However, with two times 60 patients and an alpha of 0.05 , our study has a power of $76 \%$ to detect a $25 \%$ difference between the two methods in the occurrence of a complication. To detect smaller differences between the methods far more patients would be needed. For example, to detect a $10 \%$ difference with $80 \%$ power and an alpha of 0.05 , over 800 patients would be necessary. Second, only a limited number of patients were amendable to follow-up, although this is a general problem with this kind of research. Third, to evaluate swallowing problems, FEES is the investigation of choice.

Although a similar proportion of patients experienced a complication, total major complications was borderline significantly higher in the GWDF group. Therefore, we conclude that single step dilatational tracheostomy appears to have a higher success rate compared with the guide wire dilating forceps. In our opinion, the single step dilatational tracheostomy tends to have less severe perioperative complications with comparable long-term outcome.

\section{Conflict of interest None.}

Open Access This article is distributed under the terms of the Creative Commons Attribution Noncommercial License which permits any noncommercial use, distribution, and reproduction in any medium, provided the original author(s) and source are credited. 


\section{References}

1. Fikkers BG, Breedveld P, Dongelmans DA, van der Hoeven JG, Schultz MJ, de Wit R (2010) Tracheostomy on the intensive care unit for adult patients. Neth J Crit Care 11:324-327

2. Groves DS, Durbin CG Jr (2007) Tracheostomy in the critically ill: indications, timing and techniques. Curr Opin Crit Care 13:90-97

3. Fikkers BG, Fransen GA, van der Hoeven JG, Briede IS, van den Hoogen FJ (2003) Tracheostomy for long-term ventilated patients: a postal survey of ICU practice in The Netherlands. Intensive Care Med 29:1390-1393

4. Kluge S, Baumann HJ, Maier C, Klose H, Meyer A, Nierhaus A, Kreymann G (2008) Tracheostomy in the intensive care unit: a nationwide survey. Anesth Analg 107:1639-1643

5. Añón JM, Escuela MP, Gómez V, Moreno A, López J, Díaz R, Montejo JC, Sirgo G, Hernández G, Martínez R (2004) Percutaneous tracheostomy: Ciaglia Blue Rhino versus Griggs' guide wire dilating forceps. A prospective randomized trial. Acta Anaesthesiol Scand 48:451-456

6. Ambesh SP, Pandey CK, Srivastava S, Agarwal A, Singh DK (2002) Percutaneous tracheostomy with single dilatation technique: a prospective, randomized comparison of Ciaglia Blue Rhino versus Griggs' guidewire dilating forceps. Anesth Analg 95:1739-1745

7. Fikkers BG, Staatsen M, Lardenoije SGGF, van den Hoogen FJA, van der Hoeven JG (2004) Comparison of two percutaneous tracheostomy techniques, guide wire dilating forceps and Ciaglia Blue Rhino: a sequential cohort study. Crit Care 8:R299-R305

8. Law RC, Carney AS, Manara AR (1997) Long-term outcome after percutaneous dilational tracheostomy. Endoscopic and spirometry findings. Anaesthesia 52:51-56

9. Sviri S, Van Heerden PV, Samie R (2004) Percutaneous tracheostomylong-term outlook, a review. Crit Care Resusc 6:280-284
10. Delaney A, Bagshaw SM, Nalos M (2006) Percutaneous dilatational tracheostomy versus surgical tracheostomy in critically ill patients: a systematic review and meta-analysis. Crit Care 10:R55

11. Fikkers BG, Briede IS, Verwiel JM, van den Hoogen FJ (2002) Percutaneous tracheostomy with the Blue Rhino technique: presentation of 100 consecutive patients. Anaesthesia 57:1094-1097

12. Fikkers BG, van Heerbeek N, Krabbe PF, Marres HA, van den Hoogen FJ (2002) Percutaneous tracheostomy with the guide wire dilating forceps technique: presentation of 171 consecutive patients. Head Neck 24:625-631

13. Fikkers BG, van der Hoeven JG (2004) Complications of percutaneous dilating tracheostomy. Reply to Dongelmans, DA; van der Lely, AJ; Tepaske, R; Schultz, MJ. Crit Care 8:397-398

14. Türkmen A, Altan A, Turgut N, Yildirim G, Ersoy A, Koksal C, Ayşe M, Kamali S (2008) Comparison of percutaneous dilatational tracheostomy with surgical tracheostomy. Middle East J Anesthesiol 19:1055-1067

15. Callanan V, Gillmore K, Field S, Beaumont A (1997) The use of magnetic resonance imaging to assess tracheal stenosis following percutaneous dilatational tracheostomy. J Laryngol Otol 111:953-957

16. Byhahn C, Wilke HJ, Halbig S, Lischke V, Westphal K (2000) Percutaneous tracheostomy: Ciaglia Blue Rhino versus the basic Ciaglia technique of percutaneous dilational tracheostomy. Anesth Analg 91:882-886

17. Byhahn C, Lischke V, Halbig S, Scheifler G, Westphal K (2000) Ciaglia Blue Rhino: a modified technique for percutaneous dilatation tracheostomy. Technique and early clinical results. Anaesthesist 49:202-206
18. Edwards SM, Williams JC (2001) Tracheal cartilage fracture with the Blue Rhino Ciaglia percutaneous tracheostomy system. Eur J Anaesthesiol 18:487

19. Patel PB, Ferguson C, Patel A (2006) A comparison of two single dilator percutaneous tracheostomy sets: the Blue Rhino and the Ultraperc. Anaesthesia 61:182-186

20. Higgins D, Bunker N, Kinnear J (2009) Follow-up of patients with tracheal ring fractures secondary to antegrade percutaneous dilational tracheostomy. Eur J Anaesthesiol 26:147-149

21. Kumar VM, Grant CA, Hughes MW, Clarke E, Hill E, Jones TM, Dempsey GA (2008) Role of routine chest radiography after percutaneous dilatational tracheostomy. Br J Anaesth 100:663-666

22. El Solh A, Okada M, Bhat A, Pietrantoni C (2003) Swallowing disorders post orotracheal intubation in the elderly. Intensive Care Med 29:1451-1455

23. Romero CM, Marambio A, Larrondo J, Walker K, Lira MT, Tobar E, Cornejo R, Ruiz M (2010) Swallowing dysfunction in nonneurologic critically ill patients who require percutaneous dilatational tracheostomy. Chest 137:1278-1282

24. Christenson TE, Artz GJ, Goldhammer JE, Spiegel JR, Boon MS (2008) Tracheal stenosis after placement of percutaneous dilational tracheotomy. Laryngoscope 118:222-227

25. Bell MD, Bodenham AR, Murphy PG, Mackenzie S (2007) Tracheal stenosis after percutaneous dilational tracheostomy. Anaesthesia 62:292-293 